\title{
Energetic particle evolution during coronal mass ejection passage from 0.3 to $1 \mathrm{AU}$
}

\author{
C. J. Joyce ${ }^{1}$, D. J. McComas ${ }^{1}$, N. A. Schwadron ${ }^{1,2}$, A. Vourlidas ${ }^{3}$, E. R. Christian ${ }^{4}$, R. L. McNutt ${ }^{3}$, C. M. S. Cohen ${ }^{5}$, \\ R. A. Leske', R. A. Mewaldt ${ }^{5}$, E. C. Stone ${ }^{5}$, D. G. Mitchell ${ }^{3}$, M. E. Hill ${ }^{3}$, E. C. Roelof ${ }^{3}$, R. C. Allen ${ }^{3}$, J. R. Szalay ${ }^{1}$,
} J. S. Rankin ${ }^{1}$, M. I. Desai ${ }^{6,7}$, J. Giacalone ${ }^{8}$, W. H. Matthaeus ${ }^{9}$, J. T. Niehof ${ }^{2}$, W. de Wet $^{2}$, R. M. Winslow ${ }^{2}$, S. D. Bale ${ }^{10,11}$, and J. C. Kasper ${ }^{12}$

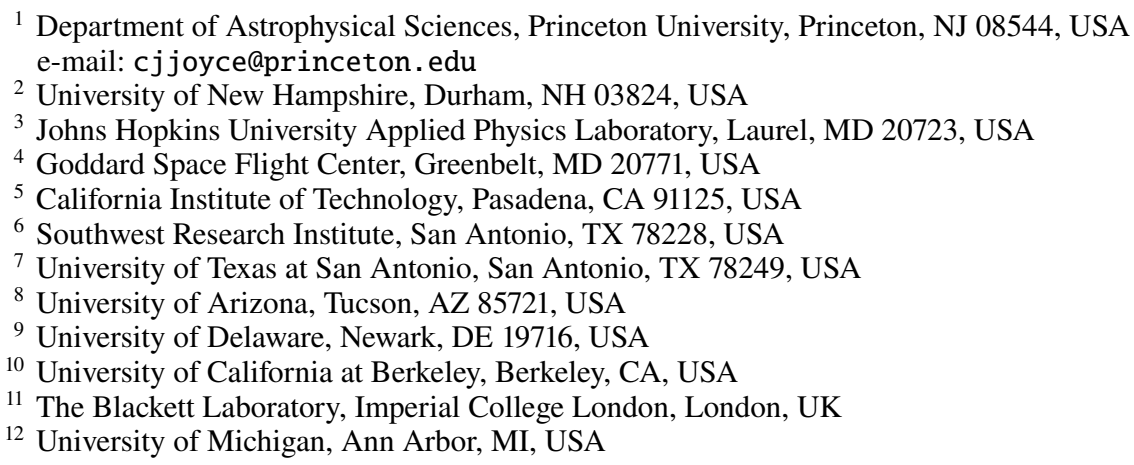

Received 17 November 2020 / Accepted 12 May 2021

\begin{abstract}
We provide analysis of a coronal mass ejection (CME) that passed over Parker Solar Probe (PSP) on January 20, 2020 when the spacecraft was at just $0.32 \mathrm{AU}$. The Integrated Science Investigation of the Sun instrument suite measures energetic particle populations associated with the CME before, during, and after its passage over the spacecraft. We observe a complex evolution of energetic particles, including a brief $\sim 2 \mathrm{~h}$ period where the energetic particle fluxes are enhanced and the nominal orientation of the energetic particle streaming outward from the Sun (from 30 to $100 \mathrm{keV} \mathrm{nuc}^{-1}$ ) abruptly reverses inward toward the Sun. This transient and punctuated evolution highlights the importance of magnetic field structures that connect the spacecraft to different acceleration sites, one of which is likely more distant from the Sun than PSP during the evolution of the CME. We discuss these characteristics and what they tell us about the source of the energetic particles. During this period, PSP was radially aligned with the Solar Terrestrial Relations Observatory A (STEREO-A), which measured the same CME when it passed 1 AU. The magnetic field measurements at both spacecraft are remarkably similar, indicating that the spacecraft are likely encountering the same portion of the magnetic structure that has not evolved significantly in transit. The energetic particle observations on the other hand, are quite different at STEREO-A, showing how transport effects have acted on the energetic particle populations and obscured the detailed properties present earlier in the development of the CME. This event provides a unique case study in how energetic particle populations evolve as CMEs propagate through the heliosphere.
\end{abstract}

Key words. acceleration of particles - solar wind - magnetic fields

\section{Introduction}

Coronal mass ejections (CMEs) are large eruptions of magnetic fields and plasma that are released from the Sun through magnetic reconnection as a result of a buildup of magnetic stress (Forbes 2000). CMEs are associated with the largest solar energetic particle (SEP) events measured at $1 \mathrm{AU}$; however, they are also capable of producing weaker events if the CME is slower or if the seed population is insufficient (Mewaldt et al. 2012). Weaker events may also be a result of the spacecraft being poorly connected to the source. The primary acceleration mechanism usually associated with CMEs is diffusive shock acceleration (DSA) within the quasi-parallel shocks that form ahead of these structures as they propagate outward from the Sun, though particle acceleration from this mechanism may also occur in weaker compressions (Giacalone et al. 2002).

Other acceleration mechanisms associated with CMEs have also been proposed, such as shock drift acceleration (SDA), which is thought to be the primary mechanism in quasiperpendicular shocks that tend to form on the western flanks of CMEs (Kallenrode 2003). SDA is typically associated with spikes in energetic particle fluxes during the passage of the shock, whereas DSA is typically associated with broader event peaks with smoother variations. Stochastic acceleration (Scholer \& Morfill 1977) is thought to occur in the turbulent regions behind shocks and may produce energetic particle spectra that differ from those typically associated with DSA (Afanasiev et al. 2014; Pallocchia et al. 2017). The magnetic reconnection 
processes that produce CMEs may also provide a source of energetic particles near the Sun that may serve as a seed population for other mechanisms. Mitchell et al. (2020) propose that a "pressure cooker" acceleration mechanism commonly associated with field-aligned current structures in planetary magnetospheres may also play a significant role in CME-associated events. They argue that similar field-aligned current structures must exist in the current sheet regions that form behind the CME flux rope structure in the low corona and they are likely to provide a consistent source of energetic particle acceleration for these events. Additionally, magnetic-island-reconnection-related processes (Zank et al. 2015; Malandraki et al. 2019) may also contribute to particle acceleration in CME-related events, driving energetic particles to higher maximum energies, and the pump acceleration mechanism (Fisk \& Gloeckler 2012) is thought to be common in the solar wind and produces consistent power law spectra of index -5 regardless of local compression conditions.

The ability to discern which of these mechanisms are most important in any given event is made difficult by the fact that most observations of CME-related SEP events have been made at $1 \mathrm{AU}$. By the time the CME reaches $1 \mathrm{AU}$, particle populations, which may have originated from multiple mechanisms, will have mixed together and been acted on by various transport processes, such as scattering, adiabatic focusing and cooling, as well as stochastic effects (Ruffolo 1995; Tylka 2001; Ng et al. 2003). The combination of these transport effects will tend to obscure characteristics that would help identify their sources. In order to get a better understanding of the mechanisms that accelerate the energetic particle populations associated with CMEs, we must make measurements much closer to the Sun, where these transport effects have minimal impact on the observed particles. In addition, multi-spacecraft comparisons between such measurements close to the Sun with those made at $1 \mathrm{AU}$ can help us to understand how transport effects act on the particle populations and determine the relative importance of acceleration that occurs as the CME is erupting from the Sun versus acceleration that occurs in transit as the CME is propagating through the heliosphere.

Launched in August of 2018, Parker Solar Probe (PSP; Fox et al. 2016) is a flagship NASA mission which endeavors to answer long standing questions about the origin of the solar wind and energetic particle events by repeatedly plunging inward toward the Sun on highly elliptical orbits over the course of an eight-year mission. Every few orbits, a Venus flyby is used to bring the spacecraft's perihelion closer to the Sun, reaching as close as 10 solar radii by the end of the mission. The Integrated Science Investigation of the Sun (IS $\odot$ IS) instrument suite (McComas et al. 2016) on PSP is designed to characterize the energetic particle environment near the Sun. IS $\odot$ IS incorporates two Enegetic Particle Instruments, EPI-Hi and EPI-Lo, designed to measure energetic particles over higher and lower energy ranges. Combined, IS $\odot$ IS is capable of measuring energetic ion fluxes from $20 \mathrm{keV}$ to $100 \mathrm{MeV}$ per nucleon. PSP/IS $\odot$ IS provides the opportunity to measure CME-related events close to the Sun where transport effects are minimal, enabling us to better discern which processes are contributing most to particle acceleration. Multi-spacecraft observations of CME events by PSP and spacecraft at $1 \mathrm{AU}$ also provide insight into how transport effects act on these energetic populations as the CME propagates outward from the Sun.

On January 20, 2020, a CME passed over PSP when the spacecraft was approximately 0.32 AU from the Sun. This event is similar in some ways to another low energy energetic particle event associated with a CME measured by PSP on
November 11, 2018 when the spacecraft was about 0.25 AU from the Sun (McComas et al. 2019; Giacalone et al. 2020; Mitchell et al. 2020). The January 2020 CME was observed remotely as it was ejected from the Sun by the Solar Terrestrial Relations Observatory A (STEREO-A), the Solar and Heliospheric Observatory (SOHO), and from the Wide-field Imager for Parker Solar PRobe (WISPR; Vourlidas et al. 2016) onboard PSP. Additionally, PSP and STEREO-A were well aligned as the CME propagated outward from the Sun and it passed over both spacecraft (separated by $\sim 5^{\circ}$ in solar ecliptic coordinates). This enables us to examine the evolution of the energetic particle populations associated with a CME as it propagates outward through the heliosphere. The event studied here provides a new and independent opportunity to examine energetic particle populations associated with a CME observed close to the Sun, providing insight into the acceleration mechanisms and the source regions that are most important early in the evolution of the CME.

\section{Event overview}

Figure 1 shows simulation results from the WSA-ENLIL+Cone model generated by the Community Coordinated Modeling Center (CCMC) as part of the Space Weather Database Of Notifications, Knowledge, Information $\left(\mathrm{DONKI}^{1}\right)$, shown to illustrate the relative positions of PSP, STEREO A, and the CME. Three times are shown: the first when the CME was ejected from the Sun, the second at the time the CME was passing over PSP, and the third when the CME was passing over STEREO-A. The simulation shows that PSP and STEREO-A were radially wellaligned at this time and both encountered the CME near its western flank. The figure also shows that the Earth was well positioned for the SOHO spacecraft to observe the CME remotely off the east limb as it was ejected from the Sun. Timing of the simulated CME arrival at PSP is in good agreement with the observations shown here, though the simulated arrival at STEREO A is somewhat earlier than the observations.

\subsection{Imaging observations of the CME}

The eruption and its source region were relatively well observed by several telescopes. We defer the detailed analysis of the imaging observations for a future paper (Vourlidas et al., in prep.). We can report, here, however, the initial findings that are relevant to our discussion. Figure 2 shows representative snapshots of the CME from STEREO-A/COR2, SOHO/LASCO-C3) and PSP/WISPR. An inspection of the low corona observations from the Solar Dynamics Observatory Atmospheric Imaging Assembly (SDO/AIA) reveals outward moving loops behind the east limb, starting at around 2:20 UT on 18 January. The eruption occurs gradually with the first set of loops leaving the AIA field of view by 5:30 UT, followed by a larger structure by 14 UT. The observations indicate that the source region is close to the central meridian of STEREO-A, however, there are no obvious signatures of the eruption. This is unsurprising, given the slow evolution of the eruption. These, so-called stealth CMEs (Robbrecht et al. 2009) are relatively common during solar minimum. Using the AIA imaging as a guide, we were able to identify few and dispersed signatures of brightenings and dimmings in the EUVI-A $195 \AA$ images (very similar to the signatures in the original stealth CME detection by Robbrecht et al. 2009) and thus make a preliminary tracing of the source region (Fig. 2).

1 https://kauai.ccmc.gsfc.nasa.gov/DONKI/view/ CMEAnalysis/15284/2 


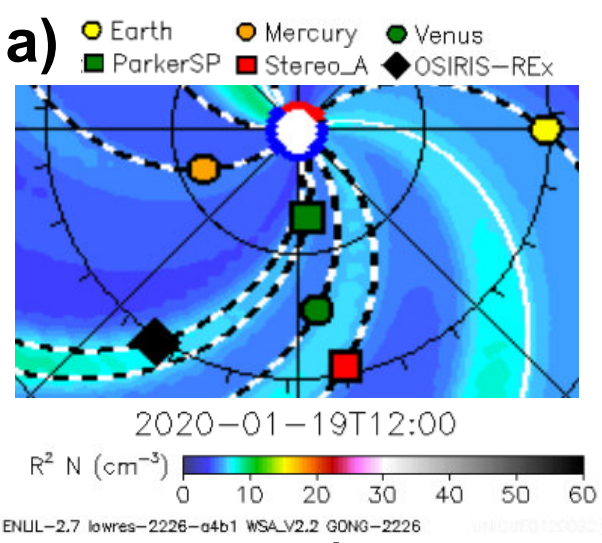

d)

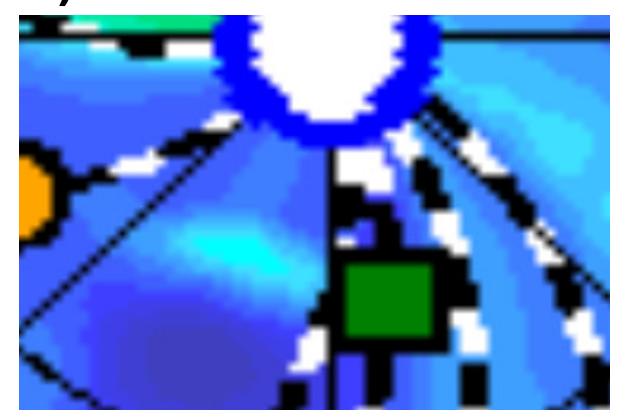

2020-01-21T06:00 b) OEarth OMercury OVenus

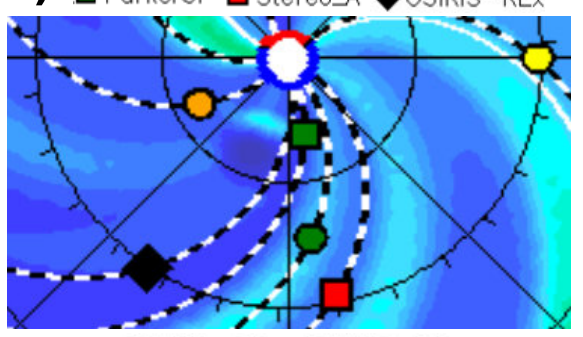

2020-01-21T06:00

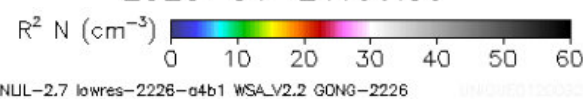

e)

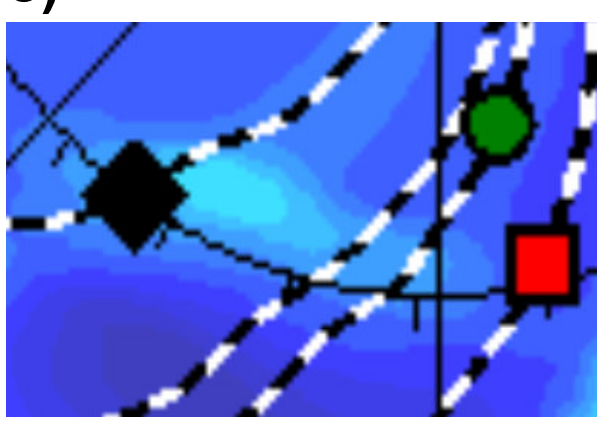

2020-01-24T00:00
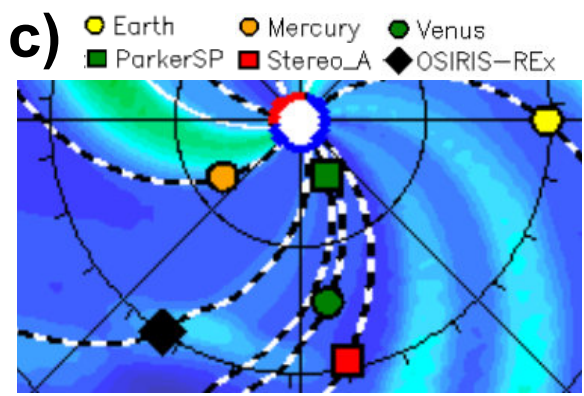

2020-01-24T00:00

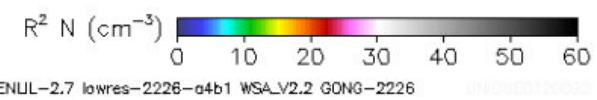

Fig. 1. Overview of the spacecraft orientation relative to the CME as it propagates outward from the Sun, using simulation results from the WSAENLIL+Cone model as provided by the Community Coordinated Modeling Center (CCMC). The location of PSP is indicated by a green square and the location of STEREO-A by a red square. During this event PSP was inbound at $\sim 0.3 \mathrm{AU}$, heading toward its fourth encounter. We see that PSP and STEREO-A are radially well-aligned during this period and both observe energetic particle enhancements as the flank of the CME passes over each spacecraft. Three snapshots in time are shown: $(a)$ when energetic particles are first measured by PSP, more than a day before the CME passes over PSP, $(b)$ late in the energetic particle event when the CME is passing over the PSP, and $(c)$ in the middle of the energetic particle event observed by STEREO-A as the CME passes over the spacecraft. Panels $d$ and $e$ are enlarged images of panels $\mathrm{b}$ and $\mathrm{c}$ to better show the position of the CME. The CME itself is not visible in panel $a$, and is somewhat faint in the other panels due to the relative weakness of the compression.
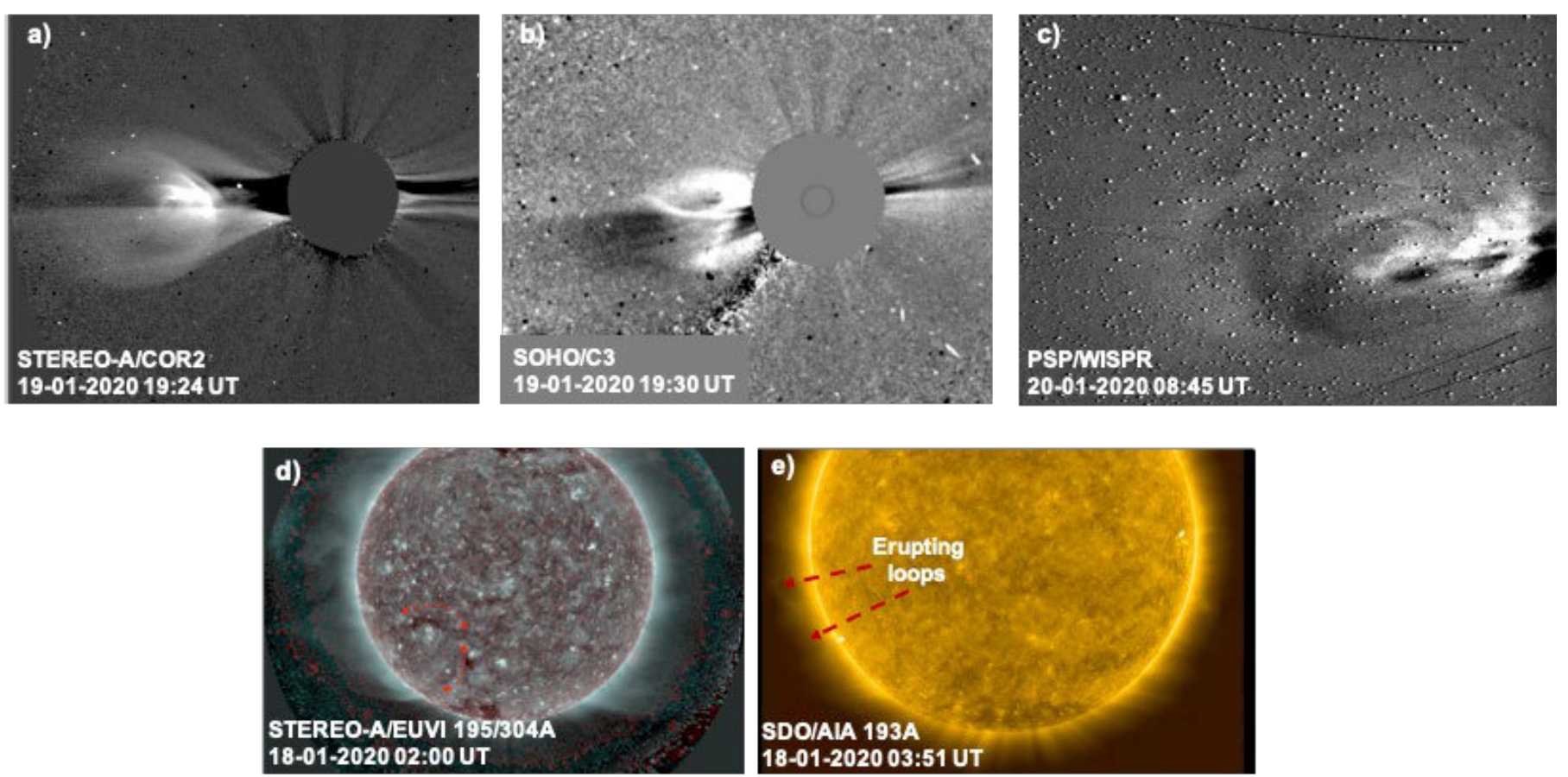

Fig. 2. Remote observations of the January $20 \mathrm{CME}$ and its likely source region. The instruments and times are shown on each panel. The red arrows demarcate the source region in EUVI, based on the erupting loops seen in AIA. The COR2 and C3 are mass images, the WISPR is a running difference image. The EUVI frame is a composite of 195 and $304 \AA$ channel wavelet-enhanced images (Stenborg et al. 2008). 


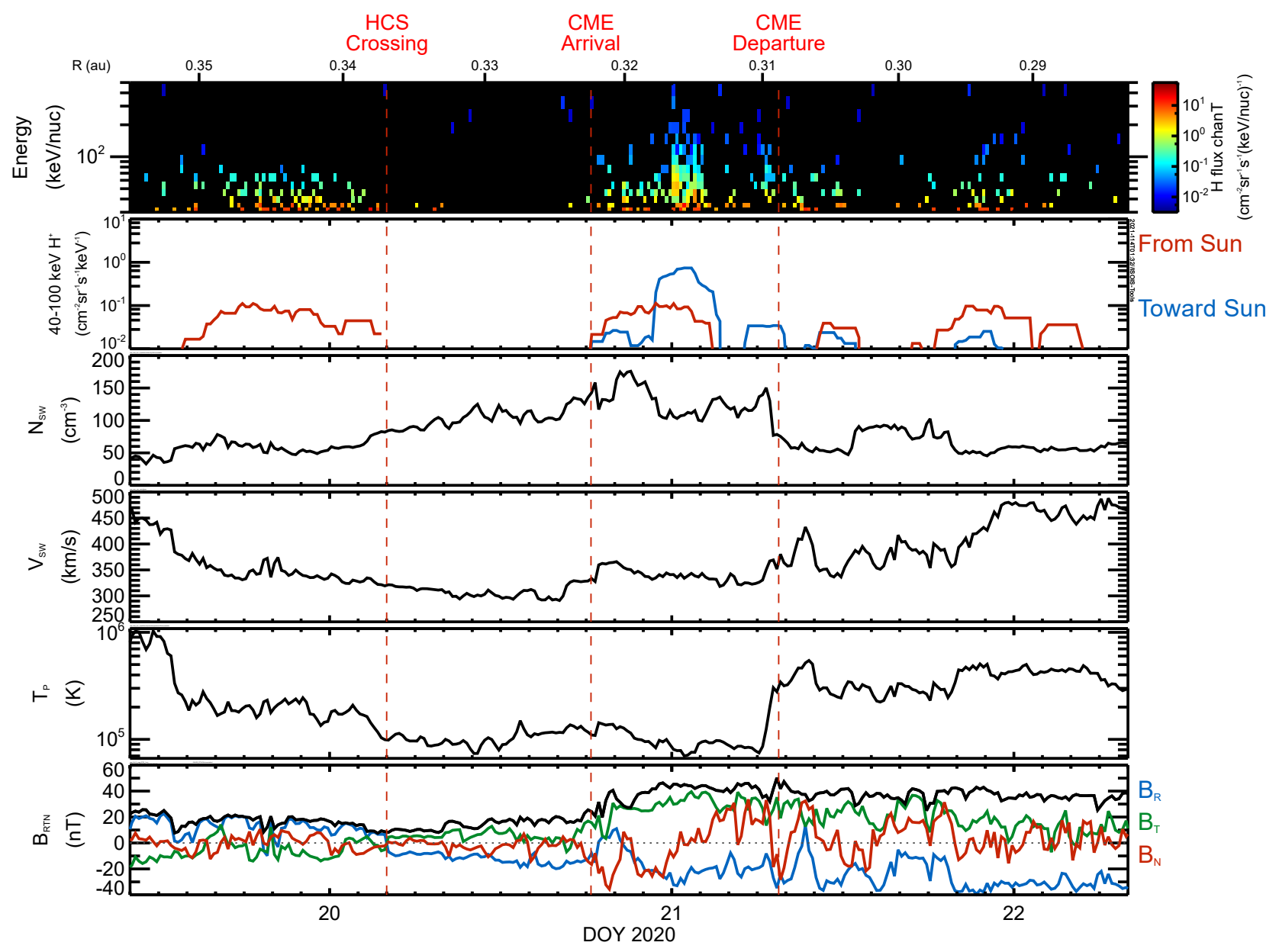

Fig. 3. Overview of the energetic particle, plasma and magnetic field lines during this event. Top panel: spectrogram of the energetic particles, showing signatures of velocity dispersion. Second panel: anisotropy of energetic particles propagating from and toward the Sun. Panels 3-5: plasma conditions as measured by the Solar Wind Electrons Alphas and Protons (SWEAP) Solar Probe Cup (SPC) (Kasper et al. 2016). Bottom panel: magnetic field in Radial, Tangential, Normal (RTN) coordinates (Fränz \& Harper 2002), as measured by the FIELDS instrument (Bale et al. 2016). On January 20, we see $B_{\mathrm{R}}$ change from positive to negative and $B_{\mathrm{T}}$ change from negative to positive, consistent with crossing the heliospheric current sheet. Estimated CME arrival and departure times are shown on the plot, bracketing a low plasma beta period, which is consistent with the passage of a magnetic cloud. Data are averaged into 15-min intervals.

It is likely that the CME originates over a wide area extending between $20-60 \mathrm{E}, 23-57 \mathrm{~S}$ of the central meridian of STEREO A $\left(67^{\circ}-107^{\circ}\right.$ and $27^{\circ}-61^{\circ}$ in Carrington longitude and latitude, respectively). This is consistent, at first glance, with the loop evolution seen in AIA and with the first results from the 3D reconstructions of the $\mathrm{CME}$ in $\mathrm{COR} 2$ and $\mathrm{C} 3$. The latter suggest that the $\mathrm{CME}$ is propagating at a $60^{\circ}$ average Carrington longitude, consistent with the Enlil simulations in Fig. 1. The LASCO height-time measurements (not shown here) suggest acceleration through 18 solar radii with the CME requiring $380 \mathrm{~km} \mathrm{sec}^{-1}$ to match the time of arrival at PSP. This is consistent with the average speed of $386 \mathrm{~km} \mathrm{sec}^{-1}$ for the $1 \mathrm{AU}$ crossing to STEREO-A. In summary, the remote sensing observations suggest that this is an extended stealth-type CME originating over a quiet Sun area, that it is accelerating throughout the corona and that PSP is crossing at the western flank.

\subsection{Energetic particle observations at PSP}

Figure 3 shows an overview of the energetic particle, plasma, and magnetic field conditions during this event. EPI-Hi was off during the period studied here due to an instrument anomaly that has since been fully resolved, so we use only data measured by
EPI-Lo. A strong energetic particle signature is first seen midway through January 19. This signature is highly anisotropic, with energetic particles mostly coming from the Sun, and shows strong evidence of velocity dispersion, with features consistent with both a remote source and one close to the Sun. The magnetic field is also nearly radial during this time, facilitating more direct transport of particles from the Sun. Interestingly, these fluxes cut out at approximately the same time that the spacecraft passes through a sector boundary crossing early on January 20, indicating that the connection to the remote particle source has been cut off by the crossing of the heliospheric current sheet.

The CME arrives at PSP at approximately 19:00 UTC on January 20. No shock is observed at the passage of the CME in the plasma data, however as noted previously, PSP is located on the western flank of the CME during its passage and it is possible that a stronger compression or even a shock is present near the nose of the CME, however no such structure is visible in the coronagraph images. It has also been established that in the absence of shocks, weaker compressions are capable of accelerating energetic particles (Giacalone et al. 2002); a particularly small compression has already been shown to accelerate such particles by PSP (McComas et al. 2019; Joyce et al. 2021). As expected, we see a significant rotation of the field relative 


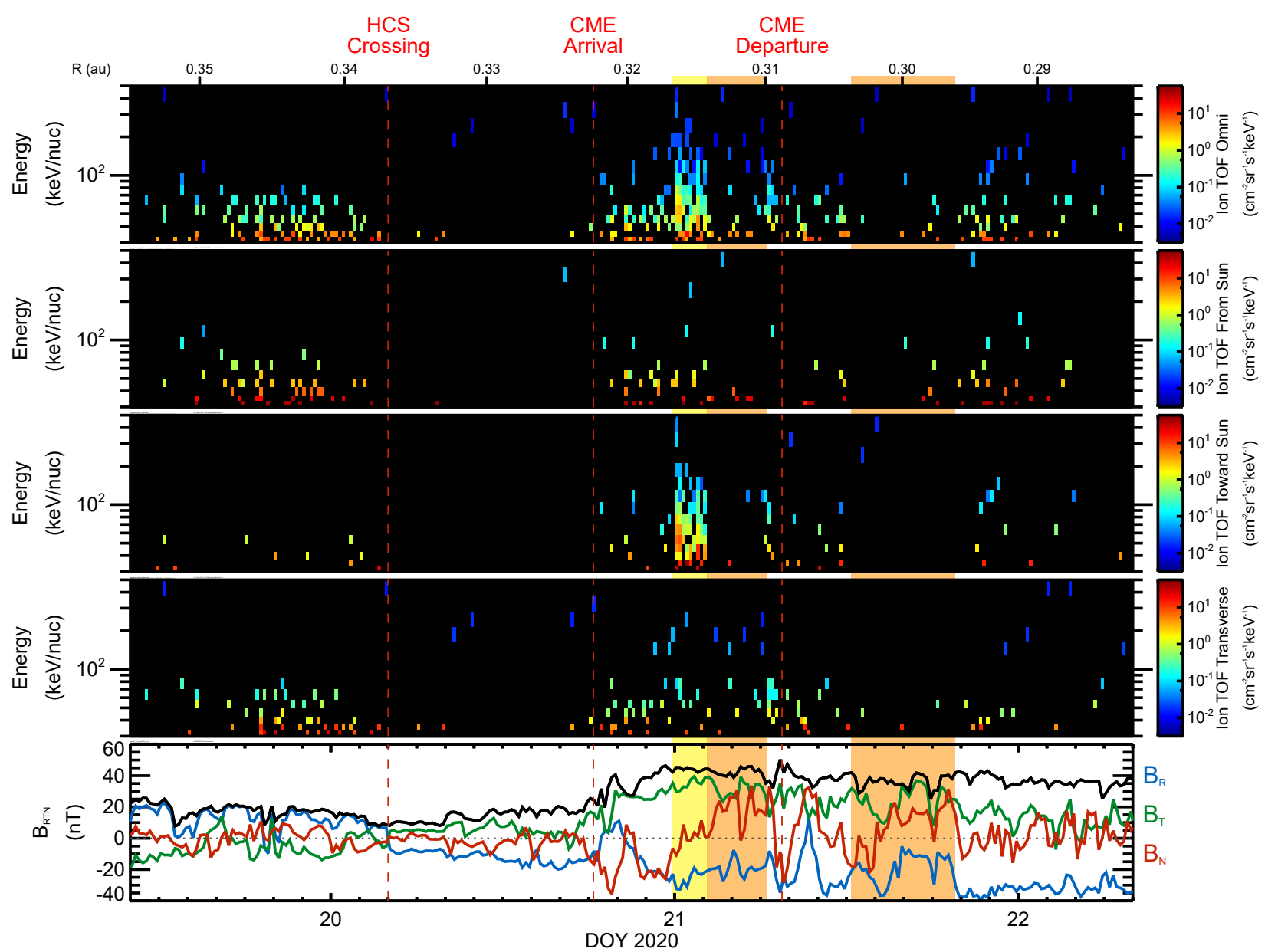

Fig. 4. Spectrograms showing the evolution of energetic particle anisotropy during the course of the event (from top to bottom: omni-directional, from Sun, toward Sun, and transverse). Particle directions are defined with respect to the sunward-facing nominal Parker spiral direction, with from-Sun being from 0 to 60 degrees, transverse from 60 to 120 degrees, and toward-Sun from 120 to 180 degrees. Details on these directions are shown in the appendix. Bottom panel: magnetic field in RTN coordinates and we see that particle anisotropies are highly correlated with changes in the magnetic field. A strong influx of energetic particles propagating toward the Sun is highlighted in yellow, while dropouts in flux are highlighted in orange. Data are averaged into 15 -min intervals.

to the undisturbed interplanetary magnetic field as the CME passes over the spacecraft. Following this rotation of the field, we observe large fluctuations in the normal component of the magnetic field. The energetic particle observations during the CME passage are characterized by complex fluctuations in the directionality of the particles as well as near complete dropouts, which are discussed in greater detail in the next section. $4 \mathrm{He}$ was also measured for this event, with intensities well below $1 \%$ that of protons. $3 \mathrm{He}$, heavier ions and electrons are not observed to be significantly above background during this period.

\section{Anisotropy and spectral analysis}

Figure 4 shows spectrograms for different look directions viewed by EPI-Lo during this period, showing the evolution of energetic particle anisotropy during the event. We see that the event is largely dominated by particles propagating outward from the Sun as well as particles moving in the transverse direction, however, we also observe interesting variations in the particle anisotropy coinciding with changes in the magnetic field, particularly the normal component. A Compton-Getting correction has not been applied to these observations since the IS $\odot$ IS pitch angle distribution data products needed for this calculation are not yet available at the time of submission. While applying a
Compton-Getting correction to these observations would affect the observations to some degree, the anisotropies shown here are large enough that such a correction would not significantly alter our qualitative assessment of the event.

As the CME passes over the spacecraft, the normal component rotates from south to north, which coincides with a very strong influx of particles moving toward the Sun (highlighted in yellow). This influx of particles lasts only a few hours and as the normal component reaches its northern apex (in RTN coordinates), the flux of particles in all directions largely drops out (highlighted in orange). This dropout lasts for a few hours until the normal component of the field spikes southward corresponding with the resumption of more isotropic energetic particle signatures. A second dropout period is seen later in the day, after the CME has departed, when the normal component of the field is again in the northward direction (also shown in orange). The magnetic field remains within the field of view (FOV) of the instrument during these dropouts, so they seem to be related to a disconnection from the source rather than the rotation of a fieldaligned beam of particles rotating out of the instrument FOV. Particles are again observed moving away from the Sun after the field rotates southward following the second dropout. Though the count rates are too low to show a clear velocity dispersion signature, the strong anisotropic signatures during the event 

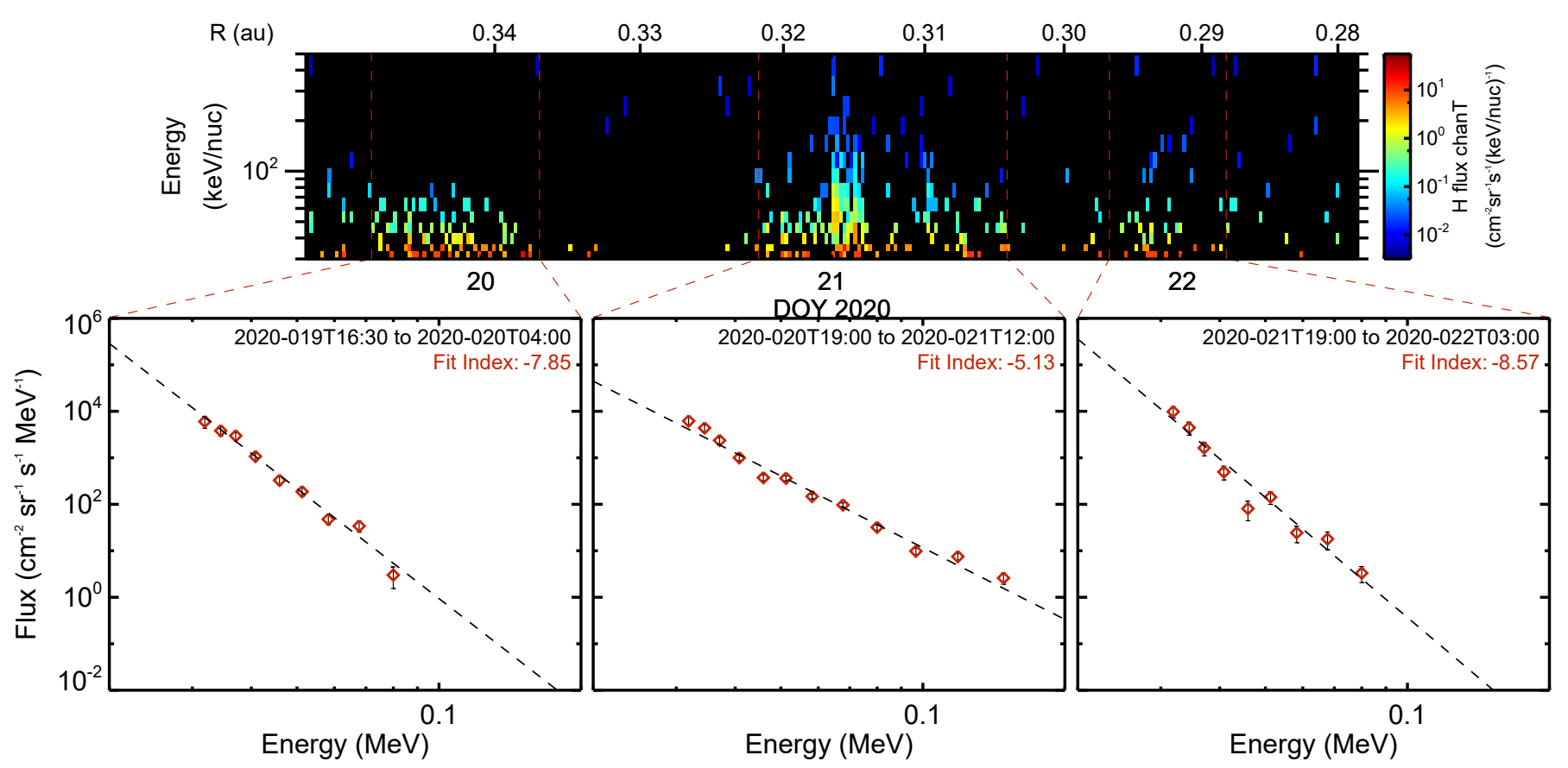

Fig. 5. Energetic particle spectra for three periods during the event, prior to, during and after the CME passage. Each spectrum is fit with a simple power law. Details on spectra computation are included in the appendix. Spectra are more than $99 \%$ protons but also include heavier ions in small amounts. The spectrogram data are averaged into 15-min intervals. Spectra uncertainties are statistical.

suggest that the dominant source of the energetic particles is remote as is discussed further in Sect. 5.

Figure 5 shows the evolution of energetic particle spectra over the course of the event. As previously stated, the spectra are mostly protons, however they also include small amounts of heavier ions. Spectra are taken for three periods where energetic particle signatures are present prior to, during and after the passage of the CME. Spectra throughout the event are consistent with a simple power law form and fits are applied to determine the spectral indices. The spectra prior to and following the passage of the CME are shown to be extremely soft, with spectral indices of about -8 , while the spectrum during the passage of the CME is somewhat harder, showing a spectral index of about -5 . SEP events with spectral indices as steep as -8 are uncommon in prior studies using data at $1 \mathrm{AU}$, though not unheard of, and it is possible that background from photons (discussed in the appendix) is artificially increasing the flux of low energy particles and making the measured spectral indices steeper than they should be, although this effect should be limited by the corrections we apply to these observations (also discussed in the appendix). Such soft spectra from early PSP observations close to the Sun are seen at other times and may be a common feature in the previously unexplored near-Sun region.

\section{Comparison with STEREO-A}

Figure 6 provides an overview of this event at STEREO-A, showing the energetic particle and magnetic field conditions during this period. Unfortunately, a high voltage anomaly prevented the collection of plasma data during the event. The magnetic field structure during the CME passage is remarkably similar to that observed by PSP when the CME was at just 0.32 AU. The field is largely tangential throughout and we see similar rotations of the normal component of the magnetic field. The energetic particles during this period are largely anisotropic coming from the
Sun, in contrast to the observations at PSP, which showed more variation, however some fluctuations of the particle anisotropy are seen in conjunction with the changes of the magnetic field. Another contrast to the observations at PSP is that we do not see a significant energetic particle signature ahead of the CMEs arrival at STEREO-A, which could be expected due to the positioning of STEREO-A on the western flank of the CME, as a nominal Parker spiral configuration at $1 \mathrm{AU}$ would not provide a magnetic connection to the CME when it was close to the Sun.

Figure 7 shows spectrograms from different look directions. This again shows that the energetic particle observations during this event have significant anisotropy, however the anisotropies remain much more consistent throughout the event than at PSP. Similar to PSP, we observe a near total dropout of particles when the normal component of the magnetic field begins to rotate from south to north, however the relative anisotropy of particles coming from the Sun and moving toward the Sun is relatively constant throughout the event. While fluctuations in the energetic particles in the north and south directions are seen with the rotation of the normal component of the magnetic field, these changes are not nearly as prominent as the variations seen at PSP. We do not find any obvious velocity dispersion signatures in the STEREO-A data for this event.

Figure 8 shows the evolution of energetic ion spectra measured by the STEREO-A Solar Electron and Proton Telescope (SEPT) during this event. Spectra are computed for three periods: the first prior to the passage of the CME, the second during the passage of the CME, and the third following the flux dropout we observe when the normal component of the field rotates from South to North. As with the spectra seen at PSP, each spectrum is well fit by a simple power law, however the spectra at STEREOA are harder overall and have higher intensities at comparable ion energies. We note that the SEPT instrument does not extend to energies quite as low as IS $\odot$ IS. In contrast to the spectra measured at PSP, there is much less variation of the spectral indices taken at different times in the event at STEREO-A. 

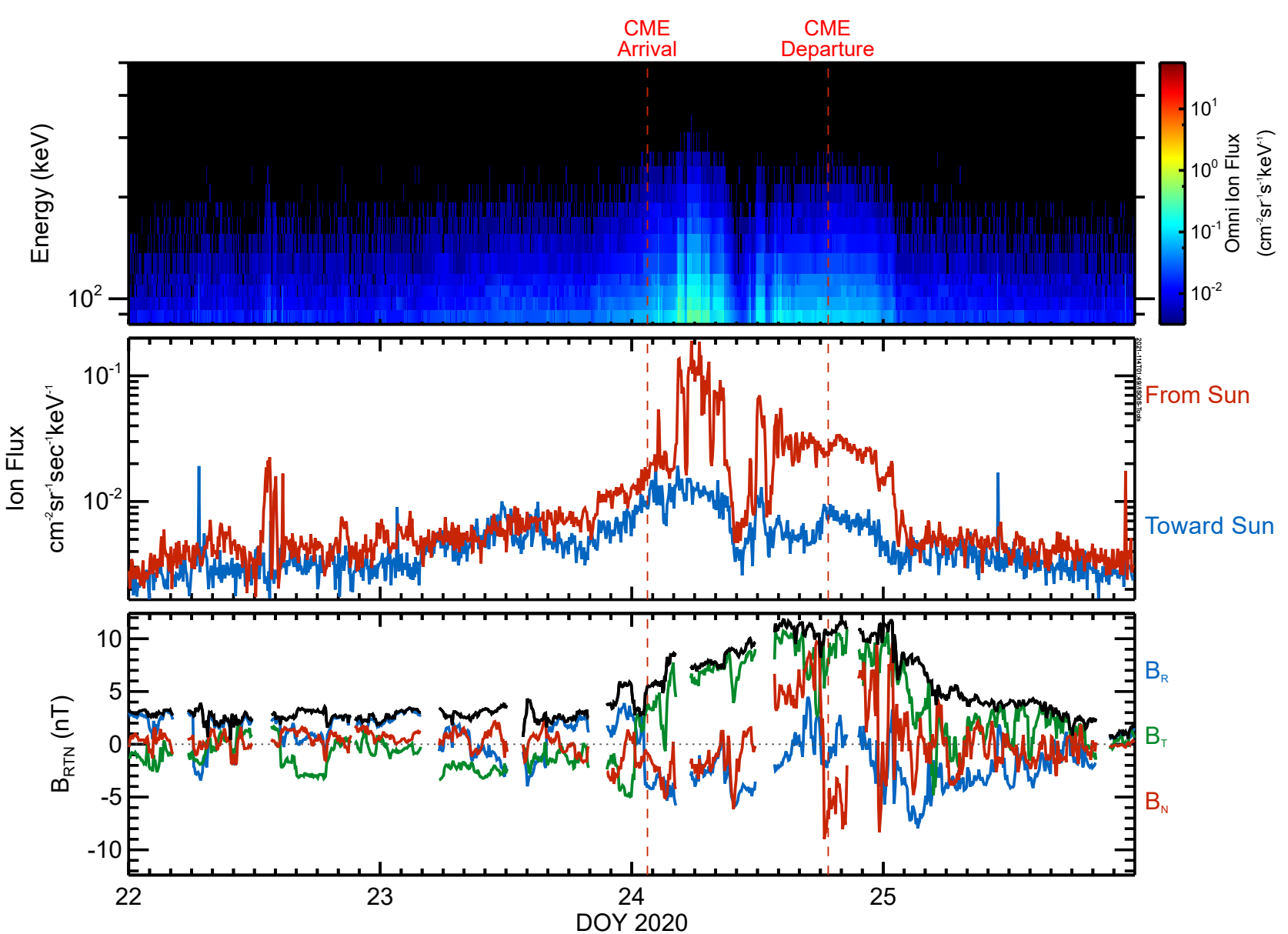

Fig. 6. Overview of event at STEREO-A, including ion data from the SEPT instrument and magnetic field data in RTN coordinates from the MAG instrument. The data are averaged into 5-min intervals.

\section{Discussion}

The event analyzed here offers a unique case study into how energetic particle populations associated with a CME change as the CME propagates outward from the Sun. The magnetic field measurements made at 0.32 AU by PSP and at 1 AU by STEREO A three days later are remarkably similar despite their spatial and temporal separation. This, combined with the close radial alignment of the spacecraft, seems to indicate that both spacecraft are encountering a very similar region of the CME. Despite the similarity of the magnetic structure seen at both spacecraft, the energetic particle observations are quite different, indicating the significance of transport effects acting on these populations.

During the course of the event, PSP saw a complex evolution of the energetic particle populations associated with the CME. Throughout the event, IS $\odot$ IS measured strong particle anisotropies which seem to correlate with variations in the magnetic field, indicating that the dominant sources of particles were remote throughout the event. PSP was located on the western flank of the CME, a region where Shock-drift acceleration typically plays a role in the acceleration at quasi-perpendicular shocks that typically form here, however since no shock is seen at PSP and the strong particle anisotropies seen throughout that seem to vary with magnetic field rotations are inconsistent with local acceleration, it is unlikely that this mechanism played a large role in this event.

Prior to the arrival of the CME at PSP, we observe an anisotropic and dispersive energetic particle population coming from the Sun while the field was nearly radial. This signature is observed while the CME was close to the Sun, about a day before it passed over PSP and while it was still accelerating, as suggested by the kinematic measurements in Sect. 2.1. No flare is observed nearby during this period, suggesting that these energetic particles are associated with the CME. While no shock is seen by the time the CME reaches PSP, it is possible that a weak shock could have formed briefly at the CME while it was close to the Sun and accelerated the particles observed during this period. It is also possible that these particles were accelerated behind the CME by reconnection processes or the "pressure cooker" process described by Mitchell et al. (2020) to sufficient energies to escape the acceleration region and propagate outward beyond the CME in field-aligned ion conics. This energetic particle signature persists for about half a day and is followed by a period that is absent of any energetic particle signatures until the CME arrives about half a day later. This dropout in particles coincides with a sector boundary crossing and it is possible crossing the heliospheric current sheet cut the spacecraft off from its magnetic connection to the remote source of particles.

The energetic particle enhancements during the CME passage over PSP are largely anisotropic and dominated by particles coming from the Sun and particles moving in the transverse direction, however we observe the strongest fluxes of the event, which extend to the highest energies, during a brief two hour period when the normal component of the field is rotating from South to North as the CME passes over the spacecraft. This period is dominated by particles streaming inward toward the 


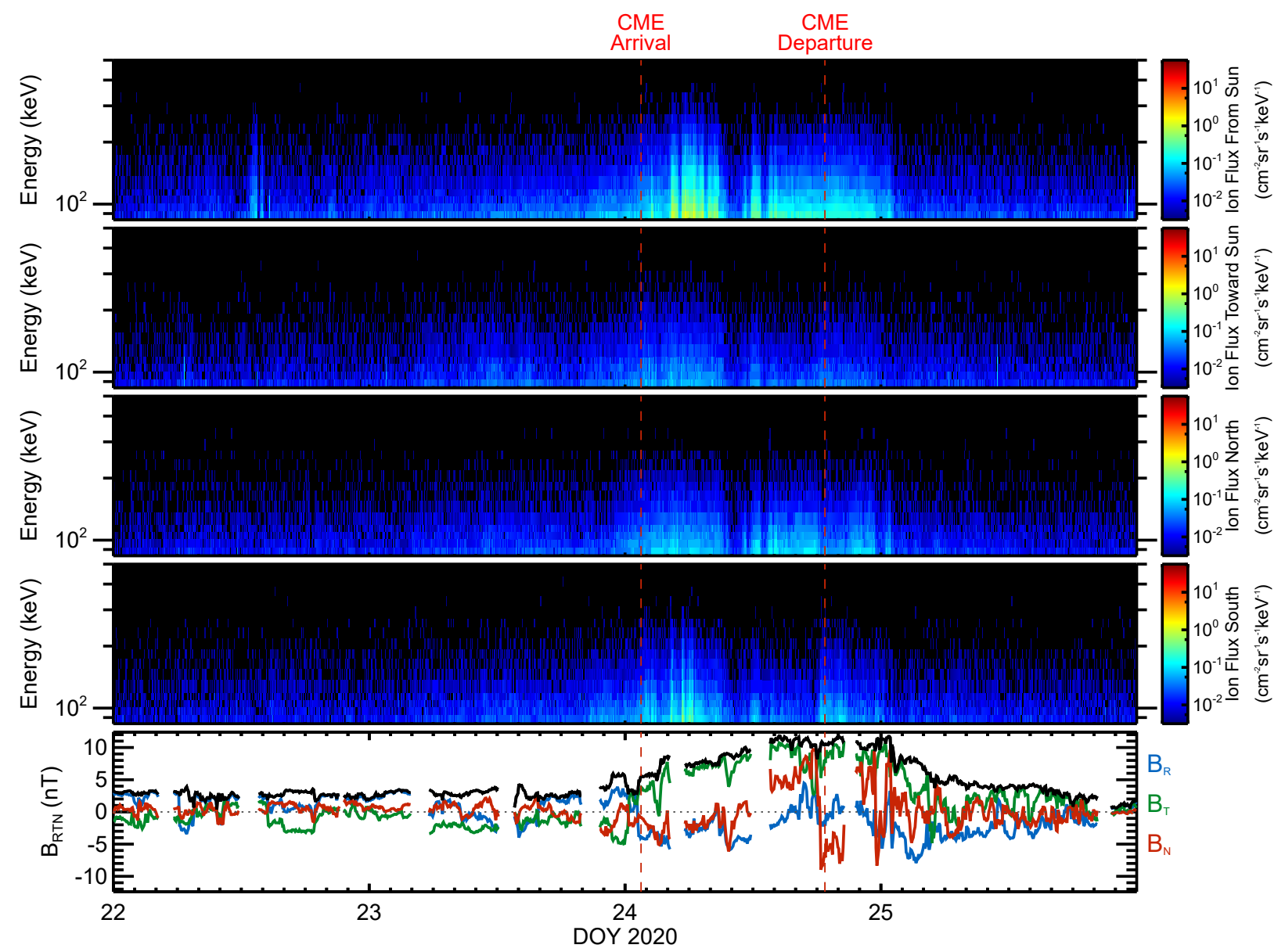

Fig. 7. STEREO-A SEPT spectrograms for energetic particles propagating from the Sun, toward the Sun and in the north and south directions, showing how particle anisotropies evolve during this period. Since plasma data are unavailable for this period, CME arrival and departure times are based purely on the magnetic field strength and are thus more speculative than those for the PSP observations. The data are averaged into 5-min intervals.

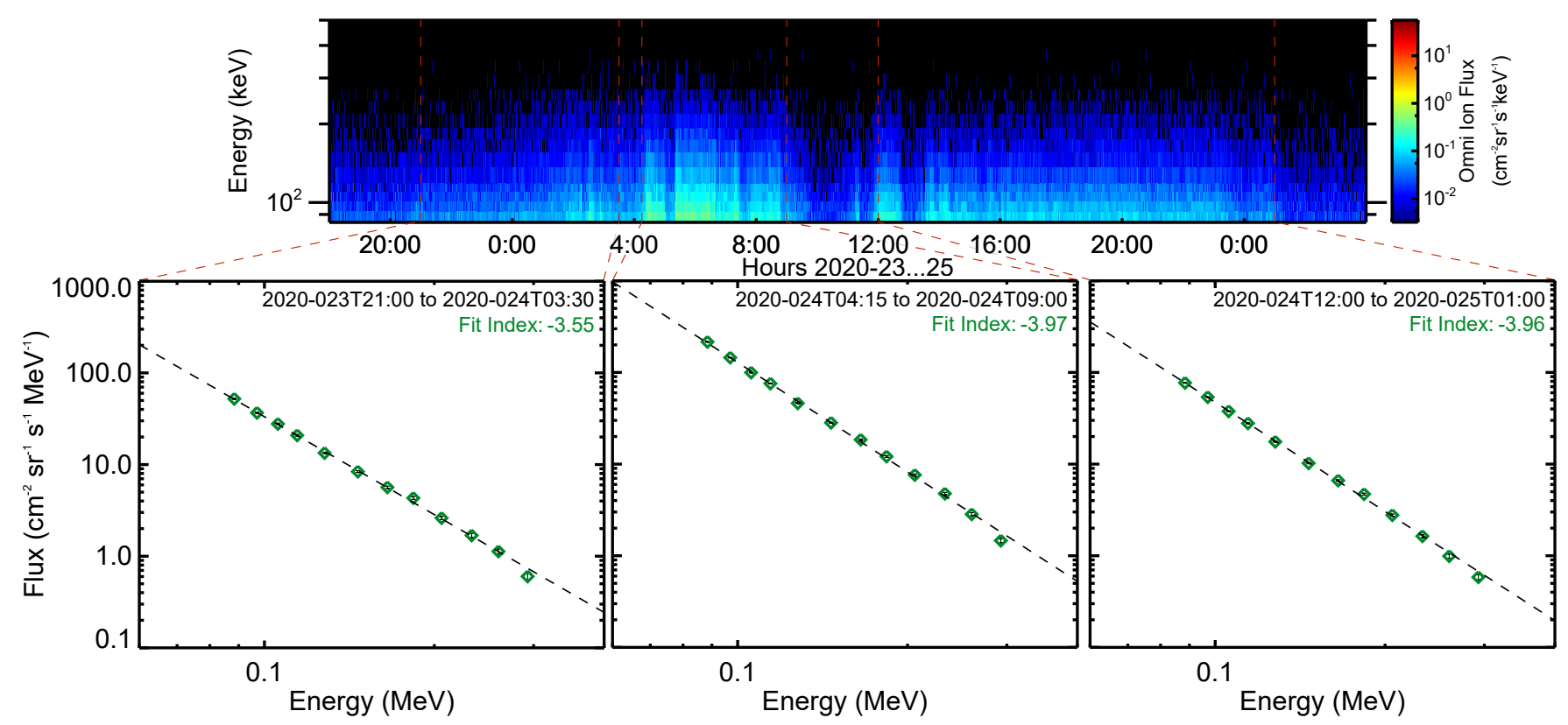

Fig. 8. STEREO SEPT spectra for three periods during the event. Each spectrum is fit to a simple power law. The spectrogram data are averaged into 5-min intervals. Spectra uncertainties are statistical. 

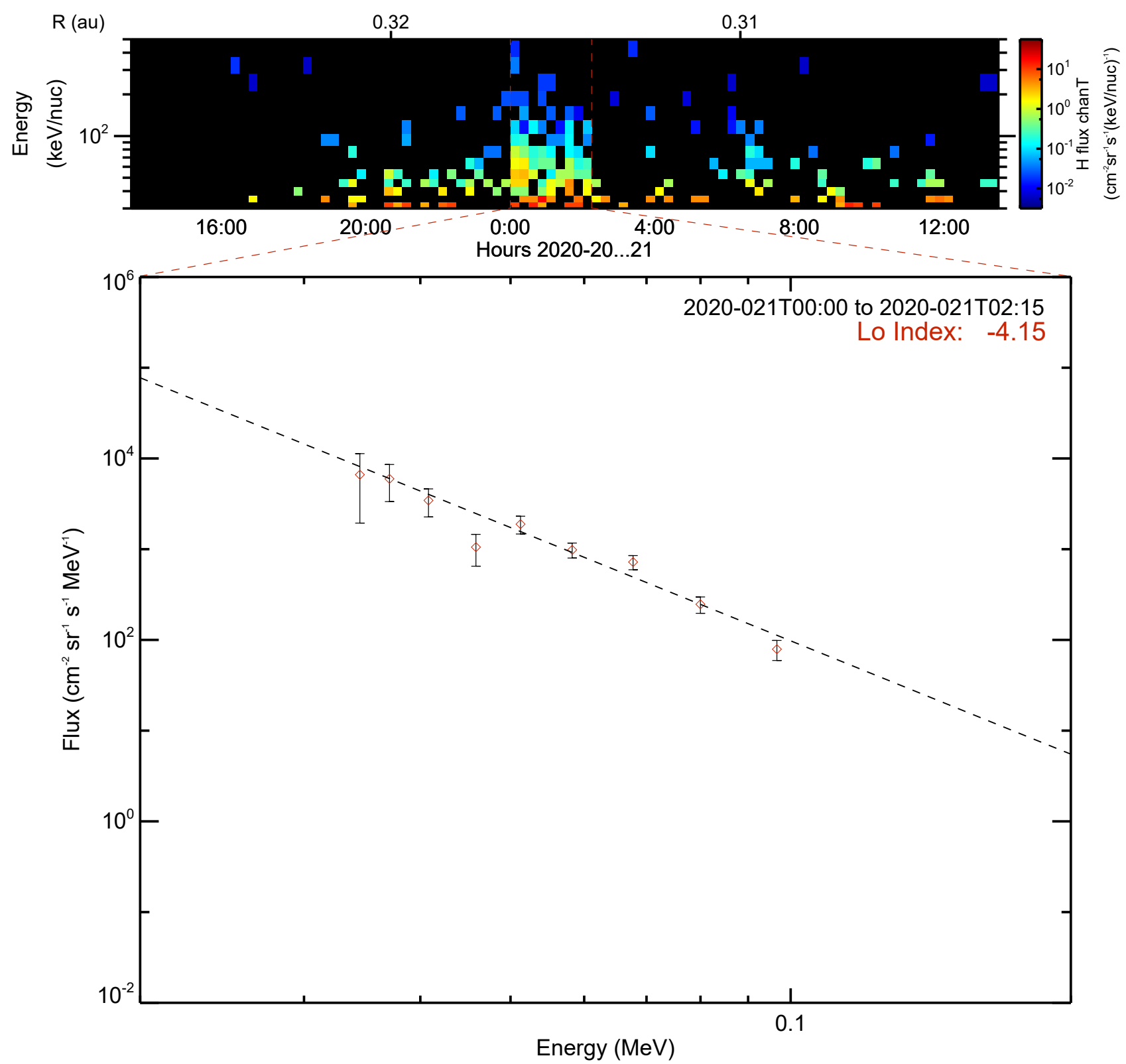

Fig. 9. Spectrum for the peak period observed by PSP, which is dominated by particles propagating toward the Sun. The spectrogram data are averaged into 15-min intervals. Spectra uncertainties are statistical.

Sun. Figure 9 shows the spectra for this period, which shows a power spectrum close to -4 , which is significantly harder than the spectrum for the rest of the event and quite similar to the spectrum observed at STEREO-A at the event's peak.

Given the abrupt change in the magnetic field orientation, particle intensity/anisotropy, and spectral characteristics, this suggests that the spacecraft is connected to a different source region during this period than for the rest of the event. One possible explanation is that PSP, located on the western flank of the CME during this time, is being connected to a stronger compression or even a shock near the nose of the CME further out from the Sun than PSP where energetic particles undergo greater acceleration. The initial CME trajectory reconstructions are consistent with this scenario.

The observations of this event at STEREO-A differ significantly compared to the measurements at PSP. Figure 10 lines up the observations at both spacecraft according to the magnetic features measured as the CME passed. The energetic particle population at STEREO-A are more uniform throughout the event in contrast to the strong variations seen at PSP. This consistency is likely the result of the mixing of different energetic particle populations as the CME propagates outward from the Sun as well as transport effects acting on the particles in transit. This demonstrates how crucial measurements made close to the Sun by PSP are in disentangling the different acceleration mechanisms that are thought to contribute to these events.

Table 1 shows a comparison between the observations of this event at PSP and STEREO-A. We note that since the fluence is computed over a range of energies measurable by both instruments, the computed PSP fluence is almost entirely from the peak period shown in Fig. 9, as most of the particles measured by IS $\odot$ IS during the event are below the energy range of the SEPT instrument. The STEREO-A observations show significantly higher event fluence and peak flux than those seen at 

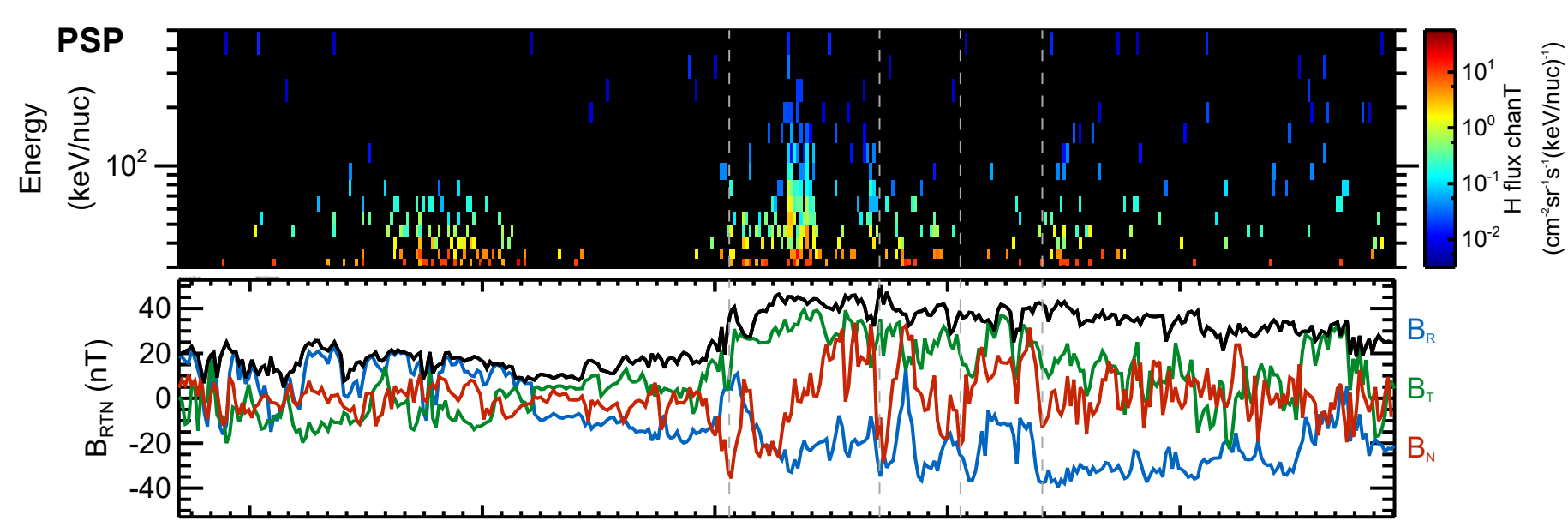

\section{$B_{T}$}

$B_{N}$
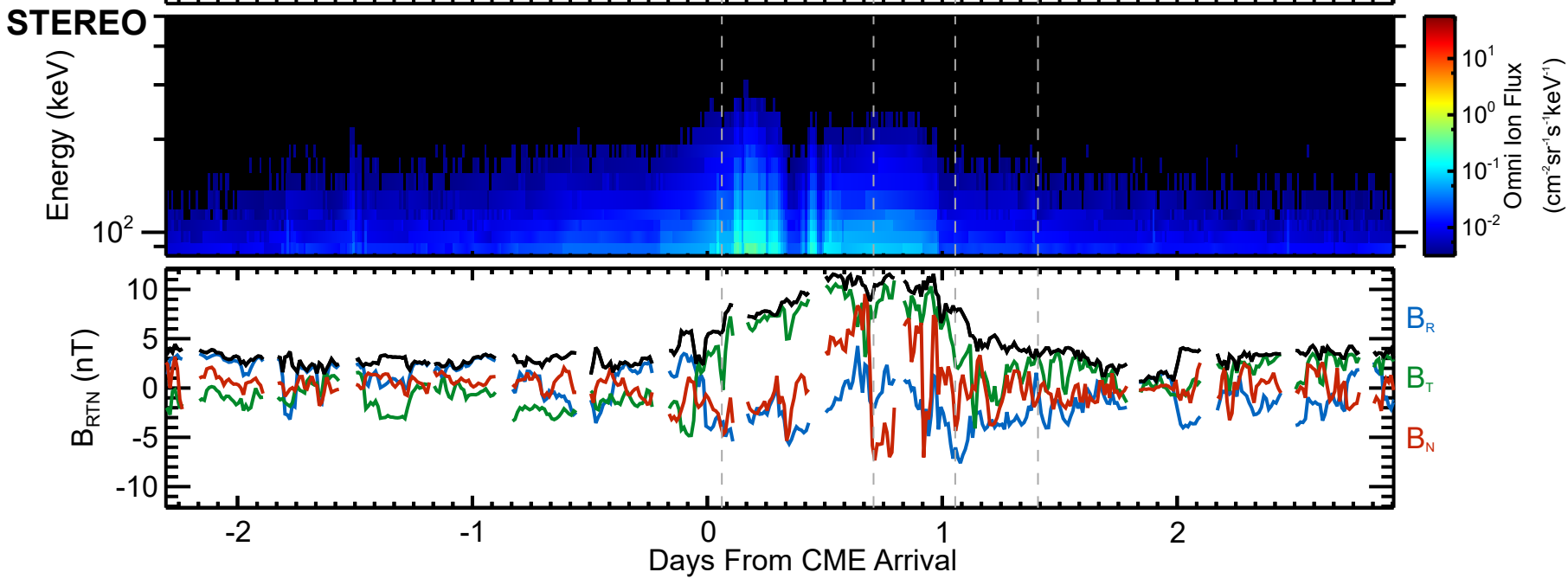

Fig. 10. Comparison between PSP and STEREO-A observations of the CME event. The data are lined up at the CME arrival and the PSP data are stretched in time by a factor of 1.3 in an attempt to match the magnetic field features between the two spacecraft. Gray dotted lines are shown to indicate points of reference used to line up these observations. The PSP data are averaged into 15-min intervals and STEREO data are averaged into 5-min intervals.

PSP. Previous observational and modeling analyses have shown the opposite: that energetic particle peak fluxes and event fluences tend to decrease with distance from the Sun (Lario et al. 2006, 2007). It is important to note, however, that these analyses studied events associated with fast CMEs with strong shock fronts that accelerate particles to much higher energies (10s of $\mathrm{MeV}$ ) than are observed here. Such events would accelerate particles across a broad shock front, however the data for this event seems to indicate that acceleration of energetic particles above $100 \mathrm{keV}$ likely occurred over a relatively small region, likely near the nose of the CME. While PSP was magnetically connected to the stronger source region for just $\sim 2 \mathrm{~h}$, by the time the CME reached $1 \mathrm{AU}$ particles from this source region would have diffused over a broader longitudinal extent, enabling measurement of elevated fluxes on the western flank over a longer period of time and thus higher event fluence. It is also possible that the higher fluxes and fluences at $1 \mathrm{AU}$ may be indicative of acceleration occurring in transit as the CME propagates outward from the Sun.

Our analysis indicates that the primary accelerator of energetic particles $>100 \mathrm{keV}$ for this event is likely diffusive shock acceleration at the nose of the CME. The observations at $0.32 \mathrm{AU}$ show that other mechanisms may also have played a role in accelerating particles while the CME was close to the Sun, possibly providing a seed population for further acceleration to
Table 1. PSP and STEREO-A comparison.

\begin{tabular}{lll}
\hline \hline & PSP & STEREO-A \\
\hline CME arrival & $2020-01-2018: 20$ & $2020-01-24$ 01:30 \\
CME departure & $2020-02107: 30$ & $2020-02418: 45$ \\
Fluence $(80-150 \mathrm{keV})$ & $4.48 \times 10^{5}$ & $4.03 \times 10^{6}$ \\
Peak flux (80-150 keV) & $2.28 \times 10^{1}$ & $1.08 \times 10^{2}$ \\
Spectral index at peak & -4.15 & -3.97 \\
\hline
\end{tabular}

Notes. Fluence is shown in units of $\mathrm{cm}^{-1} \mathrm{sr}^{-1} \mathrm{MeV}^{-1}$. Peak flux is the maximum hourly flux in these periods and is in units of $\mathrm{cm}^{-1} \mathrm{sr}^{-1} \mathrm{~s}^{-1} \mathrm{MeV}^{-1}$.

higher energies through DSA. By the time the CME reached $1 \mathrm{AU}$, the energetic particle populations are much more uniform, suggesting a single common acceleration source and obscuring other mechanisms that may have played a role closer to the Sun. Detailed modeling work is required to reconstruct the magnetic field structure of the CME and to simulate different acceleration sources in order to determine which mechanisms may have played a role in producing the energetic particle populations seen at $0.3 \mathrm{AU}$, however this is beyond the scope of this work. Future analysis of this event, modeling the propagation of the CME, will also help to better understand how various transport 
processes act on the energetic particles to produce such contrasting observations at 0.3 and $1 \mathrm{AU}$.

Acknowledgements. Parker Solar Probe was designed, built, and is now operated by the Johns Hopkins Applied Physics Laboratory as part of NASA's Living with a Star (LWS) program (contract NNN06AA01C). The IS $\odot$ IS data and visualization tools are available to the community at: https://spacephysics. princeton.edu/missions-instruments/isois; data are also available via the NASA Space Physics Data Facility (https://spdf.gsfc.nasa.gov/) Simulation results have been provided by the Community Coordinated Modeling Center at Goddard Space Flight Center through their public Runs on Request system (http://ccmc.gsfc.nasa.gov). The ENLIL Model was developed by D. Odstrcil at the University of Colorado at Boulder. We thank the STEREO team for making the SEPT and MAG data used in this study publicly available. The SOHO/LASCO data used here are produced by a consortium of the Naval Research Laboratory (USA), Max-Planck-Institut fuer Aeronomie (Germany), Laboratoire d'Astronomie (France), and the University of Birmingham (UK). SOHO is a project of international cooperation between ESA and NASA. R.M.W. acknowledges support from NASA grant 80NSSC19K0914.

\section{References}

Afanasiev, A., Vainio, R., \& Kocharov, L. 2014, ApJ, 790, 36

Bale, S. D., Goetz, K., Harvey, P. R., et al. 2016, Space Sci. Rev., 204, 49

Fisk, L. A., \& Gloeckler, G. 2012, Space Sci. Rev., 173, 433

Forbes, T. G. 2000, J. Geophys. Res., 105, 23153

Fox, N. J., Velli, M. C., Bale, S. D., et al. 2016, Space Sci. Rev., 204, 7

Fränz, M., \& Harper, D. 2002, Planet. Space Sci., 50, 217
Giacalone, J., Jokipii, J. R., \& Kóta, J. 2002, ApJ, 573, 845

Giacalone, J., Mitchell, D. G., Allen, R. C., et al. 2020, ApJS, 246, 29

Hill, M. E., Mitchell, D. G., Andrews, G. B., et al. 2017, J. Geophys. Res. Space Phys., 122, 1513

Hill, M. E., Mitchell, D. G., Allen, R. C., et al. 2020, ApJS, 246, 65

Joyce, C. J., McComas, D. J., Christian, E. R., et al. 2020, ApJS, 246, 41

Joyce, C. J., McComas, D. J., Schwadron, N. A., et al. 2021, A\&A, 650, L5

Kallenrode, M. B. 2003, J. Phys. G Nucl. Phys., 29, 965

Kasper, J. C., Abiad, R., Austin, G., et al. 2016, Space Sci. Rev., 204, 131

Lario, D., Kallenrode, M. B., Decker, R. B., et al. 2006, ApJ, 653, 1531

Lario, D., Aran, A., Agueda, N., \& Sanahuja, B. 2007, Adv. Space Res., 40, 289

Malandraki, O., Khabarova, O., Bruno, R., et al. 2019, ApJ, 881, 116

McComas, D. J., Alexander, N., Angold, N., et al. 2016, Space Sci. Rev., 204, 187

McComas, D. J., Christian, E. R., Cohen, C. M. S., et al. 2019, Nature, 576, 223 Mewaldt, R. A., Mason, G. M., \& Cohen, C. M. S. 2012, AIP Conf. Ser., 1500, 128

Mitchell, D. G., Giacalone, J., Allen, R. C., et al. 2020, ApJS, 246, 59

Ng, C. K., Reames, D. V., \& Tylka, A. J. 2003, ApJ, 591, 461

Pallocchia, G., Laurenza, M., \& Consolini, G. 2017, ApJ, 837, 158

Robbrecht, E., Patsourakos, S., \& Vourlidas, A. 2009, ApJ, 701, 283

Ruffolo, D. 1995, ApJ, 442, 861

Scholer, M., \& Morfill, G. 1977, Contributed Papers to the Sstudy of Traveling Interplanetary Phenomena (Tel Aviv, Israel: Proceedings of COSPAR Symposium B), 221

Stenborg, G., Vourlidas, A., \& Howard, R. A. 2008, ApJ, 674, 1201

Tylka, A. J. 2001, J. Geophys. Res., 106, 25333

Vourlidas, A., Howard, R. A., Plunkett, S. P., et al. 2016, Space Sci. Rev., 204, 83

Zank, G. P., Hunana, P., Mostafavi, P., et al. 2015, ApJ, 814, 137 


\section{Appendix A: Details on instrument and spectra computation}

Table A.1. Quiet times used in study.

\begin{tabular}{cc}
\hline \hline Start time & End time \\
\hline 2018-296T14:00:00 & 2018-314T16:00:00 \\
2018-325T08:00:00 & 2018-327T00:00:00 \\
2019-007T00:00:00 & 2019-011T12:00:00 \\
2019-023T00:00:00 & 2019-026T15:00:00 \\
2019-041T08:00:00 & 2019-044T18:00:00 \\
2019-052T02:00:00 & 2019-054T12:00:00 \\
2019-056T00:00:00 & 2019-057T10:00:00 \\
2019-068T07:00:00 & 2019-073T00:00:00 \\
2019-074T12:00:00 & 2019-076T12:00:00 \\
2019-085T02:00:00 & 2019-092T06:00:00 \\
2019-139T02:00:00 & 2019-143T06:00:00 \\
2019-152T06:00:00 & 2019-167T10:00:00 \\
2019-181T09:00:00 & 2019-191T18:00:00 \\
2019-235T05:00:00 & 2019-237T10:00:00 \\
2019-248T01:00:00 & 2019-252T17:00:00 \\
2019-254T08:00:00 & 2019-256T21:00:00 \\
2019-257T12:00:00 & 2019-259T00:00:00 \\
2019-260T09:00:00 & 2019-261T17:00:00 \\
2019-266T00:00:00 & 2019-268T00:00:00 \\
2019-278T00:00:00 & 2019-286T00:00:00 \\
2019-291T00:00:00 & 2019-294T00:00:00 \\
2019-305T00:00:00 & 2019-316T12:00:00 \\
2019-345T00:00:00 & 2019-350T00:00:00 \\
2019-360T00:00:00 & 2020-018T00:00:00 \\
2020-037T00:00:00 & 2020-046T00:00:00 \\
2020-053T00:00:00 & 2020-086T00:00:00 \\
2020-088T00:00:00 & 2020-105T00:00:00 \\
2020-109T00:00:00 & 2020-136T00:00:00 \\
2020-138T00:00:00 & 2020-142T00:00:00 \\
2020-171T00:00:00 & 2020-182T00:00:00 \\
\hline
\end{tabular}

Notes. These times are used to compute an overall average background spectrum, which is then scaled by a reference background time and subtracted from the event spectra. These are the same times used in Joyce et al. (2021).

The EPI-Lo instrument is a time of flight (TOF) mass spectrometer designed to measure ions from $20 \mathrm{keVnuc}^{-1}$ to $10 \mathrm{MeV} n u c^{-1}$ (McComas et al. 2016; Hill et al. 2017). The instrument includes 80 apertures that provide coverage for half the sky, enabling detailed analysis of particle anisotropies. In addition to TOF measurements, the instrument includes solid state detectors (SSDs) which measure energy deposited by particles, providing triple-coincidence data that use on board instrument processing to significantly reduce background in the observations at the cost of higher energy thresholds and somewhat lower sensitivity compared to the TOF-only data. These data were used in the course of this analysis to determine that the event was dominated by protons, but is not used in any of the plots. Because the event analyzed here is relatively weak, TOF-only data are used to generate all of the plots in order to maximize statistics.

Sources of background in the TOF-only data include ultraviolet photons and cosmic rays, both of which can trigger starts or stops within the instrument, thereby resulting in a low counting rate of accidentals (Hill et al. 2020). Cosmic rays can also create cross-talk between the detectors likewise producing a background of false events in the higher energy ranges. Efforts are currently underway to modify data processing both on board and on the ground in order to reduce the background in the data. In the course of this effort, it was discovered that certain apertures produce more background than others and we have not used those apertures in this analysis. The full list of apertures used the spectrograms and time series in Figs. 3-5 are as follows: 0, 1, 2, $3,4,5,10,11,12,13,14,15,20,21,22,23,24,30,32,33,40,41$, $42,43,45,50,51,52,53,54,55,60,61,62,63,64,65,70,71$, $72,73,74$, and 75 . During the time period analyzed here, PSP was executing rolling maneuvers and we cannot use fixed sets of apertures to determine the energetic particle anisotropy as we are able to when the spacecraft is in a nominal orientation. Three directions are defined with respect to the nominal Parker spiral direction pointing in the sunward direction: from-Sun is from 0 60 degrees, toward-Sun is from 60-120 degrees, and transverse is from 120-180 degrees. At each time step the apertures that fall within these angular bounds based on the current spacecraft orientation are averaged to determine the directional fluxes.

In order to accurately determine the spectra for this event, we must remove these backgrounds from the computed spectra. We apply the same background correction method used by Joyce et al. (2020). The background spectrum measured by IS $\odot$ IS remains essentially consistent in form over the course of the mission, with only small changes in the overall magnitude of the spectrum changing in time, however a clear pattern for this variation has not yet been established. We compute the average background spectrum using quiet times over the course of the mission (shown in Table A.1), and then scale it by the ratio of the average flux summed over all energies during a reference time near the event (for this event we use the time period from 2020-016 00:00 to 2020-019T08:00) to the average flux of the total quiet time spectrum. The spectra shown in Fig. 5 are omnidirectional using all of the apertures of the instrument except 25 , $31,34,35$ and 44 , which produce the highest rates of photoninduced accidentals. In this analysis, we find that the spectra are not significantly improved by restricting the apertures to the same degree as the spectrograms and time series. Background is removed from the STEREO spectra shown in Fig. 8 by subtracting the average fluxes from a quiet three day period just prior to the event onset, spanning January 18-21. 